\title{
Video Article \\ Obtaining High Quality RNA from Single Cell Populations in Human Postmortem Brain Tissue
}

\author{
Charmaine Y. Pietersen ${ }^{1}$, Maribel P. Lim ${ }^{1}$, Tsung-Ung W. Woo ${ }^{1,2,3}$ \\ ${ }^{1}$ Department of Structural and Molecular Neuroscience, McLean Hospital \\ ${ }^{2}$ Department of Psychiatry, Harvard Medical School \\ ${ }^{3}$ Department of Psychiatry, Beth Israel Deaconess Medical Center
}

Correspondence to: Charmaine Y. Pietersen at cpietersen@mclean.harvard.edu

URL: https://www.jove.com/video/1444

DOI: doi:10.3791/1444

Keywords: Neuroscience, Issue 30, Postmortem, microarrays, RNA, superior temporal gyrus, laser-capture microdissection, pyramidal neurons

Date Published: $8 / 6 / 2009$

Citation: Pietersen, C.Y., Lim, M.P., Woo, T.U. Obtaining High Quality RNA from Single Cell Populations in Human Postmortem Brain Tissue. J. Vis. Exp. (30), e1444, doi:10.3791/1444 (2009).

\section{Abstract}

We proposed to investigate the gray matter reduction in the superior temporal gyrus seen in schizophrenia patients, by interrogating gene expression profiles of pyramidal neurons in layer III. It is well known that the cerebral cortex is an exceptionally heterogeneous structure comprising diverse regions, layers and cell types, each of which is characterized by distinct cellular and molecular compositions and therefore differential gene expression profiles. To circumvent the confounding effects of tissue heterogeneity, we used laser-capture microdissection (LCM) in order to isolate our specific cell-type i.e pyramidal neurons.

Approximately 500 pyramidal neurons stained with the Histogene staining solution were captured using the Arcturus XT LCM system. RNA was then isolated from captured cells and underwent two rounds of T7-based linear amplification using Arcturus/Molecular Devices kits. The Experion LabChip (Bio-Rad) gel and electropherogram indicated good quality a(m)RNA, with a transcript length extending past 600 nt required for microarrays. The amount of mRNA obtained averaged $51 \mu \mathrm{g}$, with acceptable mean sample purity as indicated by the A260/280 ratio, of 2.5 . Gene expression was profiled using the Human X3P GeneChip probe array from Affymetrix.

\section{Video Link}

The video component of this article can be found at https://www.jove.com/video/1444/

Protocol

\section{1) Tissue sectioning:}

We obtained the necessary tissue (control $n=9$, schizophrenia $n=9$ ) from the Harvard Brain Tissue Resource Center, matched for age, sex and postmortem interval (PMI) (Table 1). Liquid nitrogen vapor blocks were approximately $3 \mathrm{~mm}$ thick were taken from Brodmann's area 42 (superior temporal gyrus).

Before beginning staining of pyramidal neurons, conditions must be optimized in order to obtain optimal tissue "lift" and high RNA quality. Tissue "lift" describes the process during laser-capture microdissection, where the neurons marked for capture adhere to the cap and are separated while the remaining tissue is left behind.

\section{Optimization:}

1. In order to reduce RNase activity, which compromises RNA quality, treat all surfaces, including the work area, sectioning blade and slides with an RNase decontamination solution, such as RNase Zap, in addition to wiping down with $100 \%$ ethanol.

2. Section the tissue with the cryostat set at $-17^{\circ} \mathrm{C}$. This reduces the temperature gradient between room temperature (slides), the cryostat, and the dry ice (see entire process below). If the temperature is below $-20^{\circ} \mathrm{C}$, the tissue sections tend to shred, rendering them unusable. Higher, and the temperature gradient increases, which can compromise RNA integrity.

3. The sections should be $8 \mu \mathrm{m}$ thick, as thicker sections will ultimately compromise tissue "lift". This thickness also enables correct identification of pyramidal neurons for capture.

4. Use plain uncharged glass slides (e.g. Histogene LCM slides) as these do not interfere with tissue "lift" during the laser-capture process. Membrane slides may also be used, but these are more suited to isolating large tissue structures, as the membrane tends to lift slightly during the laser-capture process, thereby increasing the infrared (IR) laser spot size and decreasing cell specificity.

5. Mount two sections per glass slide, as more than two sections per slide could compromise RNA quality due to the constant variation in temperature during the sectioning process. 
6. After sectioning, place the slides immediately into a micro slide box on dry ice in order to preserve RNA integrity. Slide boxes containing the brain sections are subsequently stored at $-80^{\circ} \mathrm{C}$ until processed.

We have previously determined that for pyramidal neurons, 500 cells per case are adequate in order to acquire enough RNA for microarray hybridization. Approximately four sections are needed per case, mainly due to sectioning and staining artifacts.

\section{2) Staining of pyramidal neurons:}

Identification of pyramidal neurons is achieved with the Histogene quick staining solution and kit, as it allows us to visualize these neurons, without prolonged exposure to aqueous solutions, thereby preserving RNA.

1. Prepare for the staining procedure by adding $1 \mu \mathrm{l}$ RNase inhibitor per $100 \mu \mathrm{l}$ of staining solution (e.g. Promega ) in order to limit the RNase activity. Four sections ( 2 slides), require $4 \mu \mathrm{l}$ of RNase inhibitor added to $400 \mu \mathrm{l}$ of the Histogene staining solution in a $1.5 \mathrm{ml}$ microcentrifuge tube (kept on ice). For each section, we use $97 \mu$ of this solution, which gives us an adequate stain to identify pyramidal neurons.

2. Prepare dehydration series and xylene: aliquot $25 \mathrm{ml}$ of the appropriate ethanols into the staining jars provided with the Histogene kit, i.e. $2 \times 75 \%, 95 \%, 100 \%$ and 2 jars of RNase-free water. Place all jars in an ice bucket, except for one $75 \%$ jar, which you place in the $-20^{\circ} \mathrm{C}$ freezer. Under a fume hood, add molecular sieves into the Xylene jar to remove excess water, which could compromise tissue "lift".

3. Switch on the waterbath (or heat block) with the temperature set to $42^{\circ} \mathrm{C}$ and place a $50 \mathrm{ml}$ Falcon tube supported by a rack in the waterbath.

4. Remove two slides from $-80^{\circ} \mathrm{C}$ and defrost them on a KimWipe for approximately 30 seconds, or just until the corners of the slides start to defrost.

5. Briefly fix the slides in the $75 \%$ ethanol for 30 seconds in the $-20^{\circ} \mathrm{C}$ freezer. Transfer the slides between staining jars using RNase-zapped forceps to reduce contamination.

6. After a 30 second wash in nuclease-free water, sections may be outlined with a PAP barrier pen in order to concentrate the solution the section, however with the plain glass slide this is not absolutely necessary.

7. Stain the four sections with the Histogene staining solution for 20 seconds, with $97 \mu$ of the stain/RNase inhibitor per section. Subsequently, dehydrate the sections in the previously prepared ethanols on ice, for 30 seconds per step. The final $100 \%$ ethanol step should be extended to 3 minutes, in order to achieve sufficient dehydration for adequate tissue "lift" and capture onto the LCM caps.

8. Immerse the slides in Xylene for 5 minutes and air-dry in a fume-hood for another 5 minutes prior to capture.

The staining steps should only be performed if laser-capturing immediately thereafter. All solutions should be changed every 4-6 slides in order to prevent contamination and to ensure proper dehydration.

\section{3) Single cell laser-capture microdissection:}

After staining, pyramidal neurons from layer III are removed using laser-capture microdissection, with the Arcturus XT system and software. Briefly, the system works by pulsing an adjustable strength IR laser through a thermoplastic film located at the base of a cap, resulting in the extension/distension of the film directly onto the cells of interest. When the cap is lifted from the tissue, the cells remain captured/captive on the cap. A pictorial summary is given in Figure 1.

Before starting this procedure, it is important to distinguish between the two types of caps available with this system. While the Macro CapSure cap is generally used for capturing larger tissues structures, the CapSure HS caps are designed to capture small numbers of individual cells and therefore these caps have a smaller surface area designated for capture. The HS caps also have rails that prevent the cap from being in direct contact with the tissue section, while the Macro cap does not. These rails reduce the effect of tissue folding that may affect or induce variability of spot size (of the laser pulse) as the Macro cap sits directly on folded, uneven tissue. For our procedure, we used the HS caps to take advantage of their ability to reduce the effect of tissue folding, but kept the Macro cap settings on the software in order to maximize the captured area as we are capturing a large number of cells.

1. Load the slides and caps onto the Arcturus XT apparatus. Use the CapSure HS caps, but keep the program setting on Macro. Click on the box "Load with overview" to obtain an overview photo of each slide.

2. Adjust the brightness/focus at $2 x$ magnification, in order to determine which section would be optimal for laser-capture. Avoid tissue sections with excessive folding, but rather choose sections that are intact, smooth, and stained well, especially near the region of interest i.e. layer III.

3. We then place a cap over the general area where we will be capturing, making sure to include layer III. Still at the $2 x$ magnification, make sure that the cap rails do not rest on any folds, as this will tilt the cap.

4. Next, we confirm the location of the IR laser spot manually at the 40x magnification. If the spot (red beam) does not correlate with the blue cross, this can be adjusted by right-clicking on the spot and selecting "located IR spot".

5. At 40x, we can begin to identify pyramidal neurons according to the following criteria (1) cells that are pyramidal in shape and (2) the proximal portion of the apical and/or basal dendrites are identifiable (Fig. 1A).

We now adjust the power and duration of the laser pulse in order to capture the pyramidal neuron. This is particularly important, as it determines the specificity of the cell captured in addition to the number of cells captured. If the pulse is too weak not all identified neurons will be captured, while if the pulse is too strong it could capture surrounding tissue/cells other than the neuron alone. Generally, with the tissue and cell specifications employed here, the laser strength $(70 \mathrm{~mW})$ and duration $(16 \mathrm{msec})$ result in a spot size of $25 \mu \mathrm{m}$, approximately in line with pyramidal neuron size (Fig. 1B). However, these settings are fine-tuned to each section in order to obtain roughly the same spot size per case. If "lift" is compromised, one can always increase the number of cells captured in order to keep the amount of RNA obtained from each case comparable. However, be sure to keep your capture time including staining under 1.5 hours, as longer than this can compromise RNA quality.

1. First save the position of the cap by clicking on the plus sign at the position function. This way, if for any reason we must remove the cap and then need to put it back on the same location, the cap will always return to precisely the same spot that you adjusted the spot size for.

2. Enter these values into the control box: 70 into power and 16 into duration. Unclick the "auto move stage" option, and make sure that the right size symbol correlates with the symbol on the panel to the right. 
3. Select the circle option (bottom right) to select a neuron that you would like to test capture, and then activate the laser by clicking on "test IR spot".

4. The spot made by the laser should firstly have a crisp dark ring around the object captured. If this ring is too light, the cell was not captured. If there is a dark spot in the middle of the dark ring, then the laser strength/duration is too great, and it might have a negative effect on the RNA present in the captured cell. Make sure that the ring is big enough to encompass the cell, but small enough that it doesn't include unwanted tissue or other cells.

5. Repeat the process on different parts of the tissue within the layer that you wish to capture, to check that the spot size does not differ depending on the location. Adjust accordingly.

6. Once the laser spot has been adjusted, continue to identify pyramidal neurons for capture. We identify approximately 500 cells per sample, which results in approximately 500pg of total RNA per sample. We also usually only use one section in order to reduce the amount of capture time, as the cap has to be readjusted for each section. Once you have identified all the cells you need, press the laser-capture button and all your cells will be automatically captured onto the cap (Fig. 1C).

7. Once all the cells have been captured, move the cap to a different part of the slide that does not contain a section. Go back to where the cells were captured and make sure that at least $90 \%$ of the neurons were removed. If not, do not try to recapture the same cells, but locate the area where most of the cells were captured, and capture more cells in the same area. Move the cap to the QC station.

8. Place the cap into the $0.5 \mathrm{ml}$ microcentrifuge tube from Applied Biosystems (cat. \# N8010611) containing 50ml of extraction buffer (PicoPure RNA Isolation kit). The cap has been designed to fit perfectly into this specific tube to prevent buffer from leaking.

9. Turn the assembly upside-down, making sure that extraction buffer covers the entire cap, and place it at the bottom of the $50 \mathrm{ml}$ Falcon tube already present in the waterbath set at $42^{\circ} \mathrm{C}$. The neurons are incubated for 30 minutes in order to remove the tissue from the cap.

10. Thereafter, centrifuge the tube and cap assembly for 2 minutes at $800 \mathrm{~g}$. Remove the cap and store the remaining cell extract at $-80^{\circ} \mathrm{C}$ until RNA extraction. As an extra precaution, you can re-examine the cap at the $\mathrm{QC}$ station on the laser-capturing apparatus to ensure that all neurons have been removed from the cap itself.

\section{4) Obtaining RNA from specific neuronal populations:}

One can go directly from capturing to isolation, or thaw the sample stored in the $-80^{\circ} \mathrm{C}$ freezer. In our experiment, we first collected all the samples needed and stored them at $-80^{\circ} \mathrm{C}$ before proceeding to RNA isolation.

RNA isolation is performed using the PicoPure Isolation Kit, which is designed to isolate small numbers of cells from LCM samples and to retain low-abundance mRNA. Generally, we follow the protocol that is included with the kit. Below, we give a basic description of the process.

1. After thawing, add $70 \%$ ethanol (provided with the kit) and centrifuge on a pre-conditioned purification column in order to bind the RNA to the column filter.

2. After washing, treat the RNA with DNase in order to ensure complete removal of DNA to eliminate the risk of DNA interference. This step is particularly important in downstream applications such as real-time qRT-PCR.

3. Following the DNA digest, there is another wash step. Check that no wash buffer remains in the column before transferring to another microcentrifuge tube, as any presence of the buffer in your final sample will result in less starting RNA for amplification. To ensure this, we centrifuge the final wash step for 2.5 minutes instead of 2 minutes as suggested in the protocol provided.

4. Finally, elution buffer is added and incubated on the filter in the column for 1 minute followed by centrifugation, in order to elute the total RNA

5. Pipette $1.3 \mathrm{ul}$ of each of the samples in separate $0.5 \mathrm{ml}$ tubes to run an Experion HighSens LabChip. Freeze the rest of the sample at $-80^{\circ} \mathrm{C}$.

Quality control is very important when isolating RNA, especially when the quantity is small and the amount needed, such as for microarray experiments $(15 \mu \mathrm{g})$, is large. The extracted RNA therefore must undergo quality control at various time points throughout the process. The first occurs after extraction. We establish the total RNA integrity via gross examination of the $18 \mathrm{~S} / 28 \mathrm{~S}$ peaks of the electropherograms and virtual gel generated by Experion HighSens LabChip. This method is quick and provides two means of measurements to check RNA quality- an electropherogram and a virtual gel (Fig. 2). If the quality of the total RNA is relatively good (Fig. 2A, B vs. C, D), we then proceed with linearly amplifying the mRNA, which accounts for roughly $2 \%$ of the total RNA.

\section{5) Linear amplification:}

Amplification of RNA extracted from laser-captured tissues is necessary in order to produce an adequate amount of RNA for subsequent microarray hybridization and qRT-PCR experiments. In order to minimize the possibility of potential confounds noted with T7-based linear amplification, we minimized the number of rounds of amplification necessary by maximizing the amount of the starting laser-captured tissue materials.

Our preliminary data (not shown) suggest that two rounds of linear amplification (RiboAmp HS ${ }^{\text {PLUS }}$ kit) of total RNA extracted from tissues that contain approximately 500 cells would produce approximately $50 \mu \mathrm{g}$ of aRNA -- sufficient for the performance of both microarray and qRT-PCR experiments. If possible, avoid unnecessary transferring of sample between $0.2 \mathrm{ml}$ and $0.5 \mathrm{ml}$ tubes by using $0.5 \mathrm{ml}$ block in the thermal cycler and the $0.5 \mathrm{ml}$ tubes provided in kit.

Again, the protocol followed is the one provided with the kit. An abbreviated version is described here.

1. Add $1 \mu \mathrm{l}$ of the primer provided to the total RNA sample (approx. $11 \mu \mathrm{l}$ ) and incubate at $65^{\circ} \mathrm{C}$ for 5 minutes, followed by chilling at $4{ }^{\circ} \mathrm{C}$ for 1 minute.

2. Add first strand cDNA components together with SuperScript III Reverse Transcriptase and incubate at $42^{\circ} \mathrm{C}$ for 1 hour

3. After second strand cDNA synthesis, purify the resulting cDNA through a purification column provided. Here we recommend that when transferring the sample plus binding buffer to the purification column, to centrifuge the sample tubes twice - once after transferring the sample to the column, as up to $1 \mu \mathrm{l}$ of sample remains on the sides of the tubes after transfer.

4. Wash the cDNA with the wash buffers provided and elute the purified cDNA with the elution buffer. 
5. Add the in vitro transcription components and incubate for 6 hours at $42^{\circ} \mathrm{C}$.

6. After a DNase step, purify the resulting aRNA with the buffers provided, eventually eluting it in a total of $12 \mu$ l of elution buffer.

The RNA undergoes another round of linear amplification (with separate primers - see kit protocol), after which more quality control steps are employed. One ul of the sample is diluted to approximately $250 \mathrm{ng} / \mathrm{ul}$ to determine the mRNA transcript length and concentration with the StdSens LabChip (Experion). Traditional Affymetrix microarray technology requires the mRNA to be at least 600 nucleotides in length in order to be detected. The gel and electropherogram obtained from the LabChip should therefore depict this minimum mRNA transcript length (Fig. 3).

An additional quality control is determined via a NanoDrop spectrophotometer analysis, where a ratio made up of two optical densities, i.e. A260 and A280, determines RNA purity. Samples with an optical density around 2.1 should be labeled for gene expression analysis, although ratios up to 2.7 have been shown to have comparable hybridization quality. The concentration should also be between $800 \mathrm{ng} / \mu \mathrm{l}-1 \mu \mathrm{g} / \mu \mathrm{l}$, as lower than this has led to RNA degradation while in storage (even at temperatures below $-80^{\circ} \mathrm{C}$ ) and has also led to poor microarray results.

\section{6) Biotin-labeling mRNA samples for Affymetrix microarray analysis:}

The TURBO Biotin labeling kit (end-labeling) from Molecular Devices is used to label the aRNA obtained from amplified samples. A shortened version of the protocol is given here.

1. Adjust the volume of $20 \mu \mathrm{g}$ of each aRNA sample to $13 \mu \mathrm{l}$ total by adding nuclease-free water or by concentrating the sample in a vacuum centrifuge, depending on the initial concentration. Even though only $15 \mu \mathrm{g}$ is necessary for the Affymetrix chip used, we add $20 \mu \mathrm{g}$ to the initial reaction, as some RNA is lost during incubation and centrifugation.

2. Incubate the biotin reaction at $85^{\circ} \mathrm{C}$ for 30 minutes, and then chill at $4^{\circ} \mathrm{C}$.

3. Remove the excess biotin label via centrifugation with the specialized column provided with the kit. In some cases, especially with degraded mRNA such as that obtained from FFPE (formalin-fixed, paraffin-embedded) samples, it might be the case that not all the mRNA is eluted in the single centrifugation step. We then recommend adding between 1-5 $\mu$ l extra nuclease-free water to the column with a subsequent extra centrifugation step of 1 minute. This ensures that most of the labeled sample is eluted.

4. The final volume is $20 \mu \mathrm{l}$, with a concentration of $1 \mu \mathrm{g} / \mu \mathrm{l}$. If extra nuclease-free water was added, concentrate the samples to the correct volume with a vacuum centrifuge. We recommend keeping the storage concentration before starting hybridization over $800 \mathrm{ng} / \mu \mathrm{l}$, as we have found that less than this will lead to degradation during storage and/or hybridization resulting in poor microarray results.

aRNA was then hybridized and gene expression profiled, using the Human X3P GeneChip probe array from Affymetrix. As this chip was designed for more degraded aRNA, we used it as a precaution, as many factors we cannot control can affect the quality of postmortem tissue. As our lab did not have the adequate facilities for hybridization, the process was performed at the Partners Genomic Core facility.

\section{Representative Results:}

\section{$1+2+3)$ Tissue sectioning, staining of pyramidal neurons and laser-capture microdissection:}

The protocol described above should result in two slides with four sections per slide. Each section should be smooth with minimal tearing, cracking and folding. With correct staining, the stain will identify darkly stained pyramidal neurons around $20-25 \mu \mathrm{m}$ in size with a pyramidal shape and visible apical dendrites. With the CapSure HS caps at Macro settings, and correct adjustment of the laser power and strength for each section, you should obtain around $500-700$ cells per section/case resulting in at least 500pg of total RNA. Approximately $85-100 \%$ of the neurons will adhere to the cap (Fig. 1).

\section{4) Obtaining RNA from specific neuronal populations:}

As described, after total RNA isolation, we check the RNA quality by means of an electropherogram and virtual gel via the Bio-Rad Experion. In the electropherogram, you should see two distinct peaks corresponding to the $18 \mathrm{~S}$ and $28 \mathrm{~S}$ ribosomal RNA units. With postmortem tissue, however, this is not always the case, as the tissue may be degraded due to factors prior to sectioning. You will normally see a large bump indicating degradation, with a large peak around 18S, and a smaller $28 \mathrm{~S}$ peak (Fig. 2A, B). As long as the electropherogram profiles are comparable across samples, we have found this guideline to result in mRNA quality good enough to perform both RT-PCR and microarray studies (Imbeaud et al., 2005).

If there is too much degradation - as indicated by a large area under the curve of the electropherogram, or if peaks are not visible (Fig. 2C, D) the cells should be captured again from the tissue. We would also recommend doing a tissue-scrape test, where RNA is extracted from whole fragments of the section (not just cells), before recapturing the cells to determine whether the tissue block itself is degraded.

\section{5) Linear amplification:}

To test the quality of the mRNA after two rounds of linear amplification, we made use of both the Bio-Rad Experion StdSens LabChip and the NanoDrop spectrophotometer. Traditional Affymetrix microarray technology requires the mRNA to be at least 600 nucleotides in length in order to be detected, which was obtained with this protocol. In fact, mRNA lengths were detected into the 1000-nucleotide range. The electropherogram also reflects this with large peaks slowly descending as a function of time (Fig. 3A, B).

The NanoDrop readings indicated an A260/A280 ratio average of 2.5 across samples, indicating viable mRNA (Table 2). 
Our results indicate that with this protocol, both the quantity and quality of RNA obtained is good enough to interrogate gene expression differences via the Affymetrix human X3P GeneChip.

\section{6) Biotin-labeling mRNA samples for Affymetrix microarray analysis:}

After hybridization to the Affymetrix human X3P GeneChip, we achieved percent calls of an average of $26.6 \%$ (Table 2), indicating adequate hybridization and probe intensities.

Table 1: Cohort summary

\begin{tabular}{|c|c|c|c|c|}
\hline Samples & Group & Sex & Age & PMI \\
\hline C1 & CONTROL & $F$ & 79 & 15.00 \\
\hline $\mathrm{C} 2$ & CONTROL & $M$ & 22 & 21.47 \\
\hline $\mathrm{C} 4$ & CONTROL & $M$ & 75 & 20.25 \\
\hline C5 & CONTROL & $M$ & 80 & 15.50 \\
\hline $\mathrm{C} 6$ & CONTROL & $F$ & 58 & 21.08 \\
\hline C8 & CONTROL & $M$ & 61 & 17.00 \\
\hline C10 & CONTROL & $F$ & 71 & 20.50 \\
\hline C11 & CONTROL & $F$ & 90 & 12.66 \\
\hline C12 & CONTROL & $F$ & 86 & 6.92 \\
\hline MEAN & & $4 \mathrm{M} / 5 \mathrm{~F}$ & 69.11 & 16.71 \\
\hline S1 & SCHIZOPH. & $F$ & 93 & 6.92 \\
\hline S2 & SCHIZOPH. & $M$ & 55 & 21.40 \\
\hline S3 & SCHIZOPH. & $F$ & 67 & 21.80 \\
\hline S4 & SCHIZOPH. & $F$ & 55 & 22.00 \\
\hline S5 & SCHIZOPH. & $M$ & 36 & 17.97 \\
\hline S6 & SCHIZOPH. & $M$ & 62 & 10.75 \\
\hline S8 & SCHIZOPH. & $F$ & 92 & 17.80 \\
\hline $\mathrm{S} 11$ & SCHIZOPH. & $M$ & 56 & 21.83 \\
\hline $\mathrm{S} 12$ & SCHIZOPH. & $F$ & 88 & 13.33 \\
\hline MEAN & & $4 \mathrm{M} / 5 \mathrm{~F}$ & 68.11 & 16.90 \\
\hline
\end{tabular}

Abbreviations: F - female, M - Male, PMI - postmortem interval, schizoph. - schizophrenia.

Table 2: Summary of results depicting RNA concentration, quality and hybridization efficiency

\begin{tabular}{|l|l|l|l|l|}
\hline Samples & [mRNA] ng/ul & A260/A280 & Probe Intensity & Percent call \\
\hline C1 & 1318.01 & 2.67 & 63 & 19.5 \\
\hline C2 & 2312.93 & 2.37 & 147 & 30.6 \\
\hline C4 & 1811.92 & 2.44 & 69 & 26.6 \\
\hline C5 & 1316.26 & 2.51 & 78 & 34.7 \\
\hline C6 & 1663.24 & 2.39 & 66 & 33.8 \\
\hline C8 & 633.05 & 2.54 & 101 & 15.2 \\
\hline C10 & 1326.4 & 2.47 & 92 & 32.5 \\
\hline C11 & 994.24 & 2.75 & 76 & 22.4 \\
\hline C12 & 817.23 & 2.7 & 56 & 15.2 \\
\hline S1 & 2560.88 & 2.47 & 78 & 25.0 \\
\hline S2 & 2169.61 & 2.43 & 76 & 26.5 \\
\hline S3 & 2067.32 & 2.52 & 87 & 22.1 \\
\hline S4 & 1563.89 & 2.75 & 82 & 28.1 \\
\hline S5 & 2071.44 & 2.44 & 88 & 28.2 \\
\hline
\end{tabular}




\begin{tabular}{|l|l|l|l|l|}
\hline S6 & 2532.59 & 2.34 & 72 & 30.7 \\
\hline S8 & 2189.22 & 2.5 & 62 & 31.8 \\
\hline S11 & 1669.89 & 2.47 & 41 & 27.3 \\
\hline S12 & 1739.35 & 2.47 & 42 & 28.0 \\
\hline MEAN & 1708.75 & 2.51 & 76.44 & 26.57 \\
\hline
\end{tabular}

\section{Figure 1: Laser-capture microdissection}

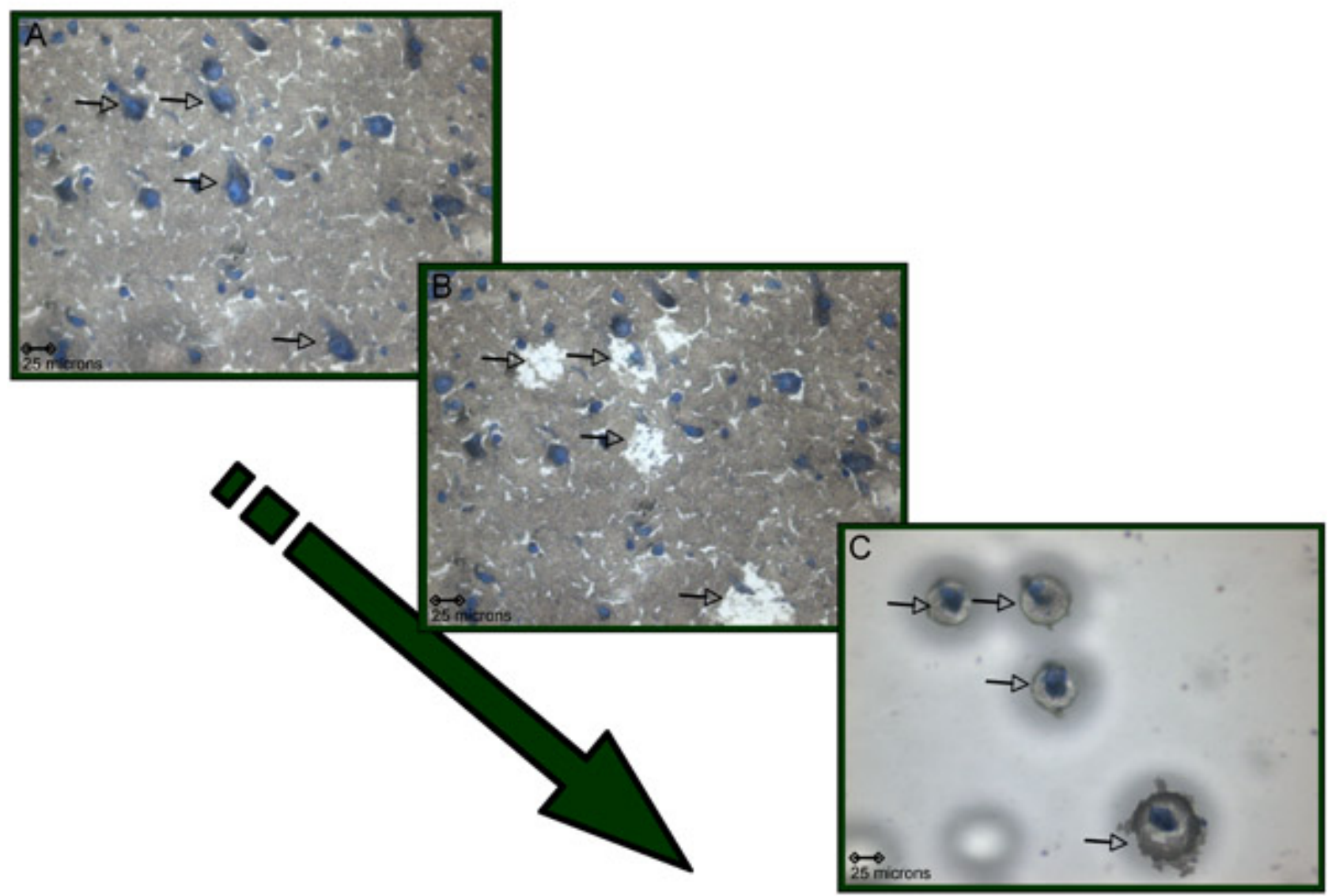

Figure 1: Summary of the laser-capture microdissection process. A) Pyramidal neurons are identified morphologically. B) After the laser has pulsed through the thermoplastic film, the cells adhere to the cap and are therefore no longer on the slide, leaving surrounding tissue behind. C) Photomicrograph of a homogeneous cell population adhering onto the CapSure Cap after laser capturing. 


\section{Figure 2: Total RNA quality control}

\section{Good quality}
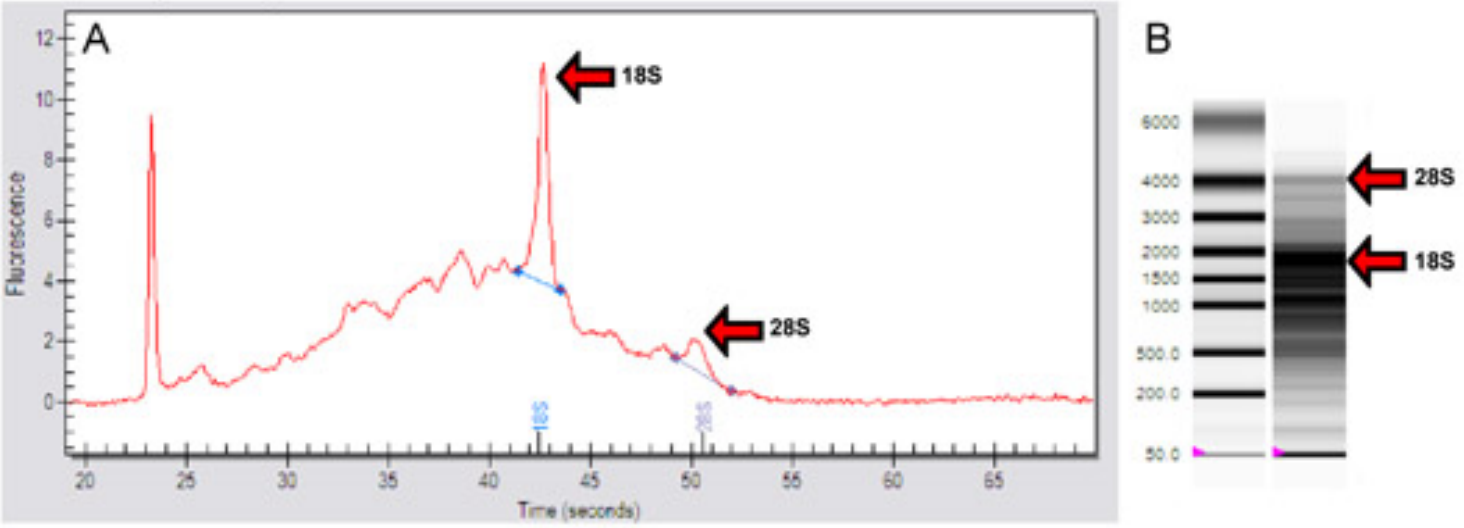

\section{Bad quality}
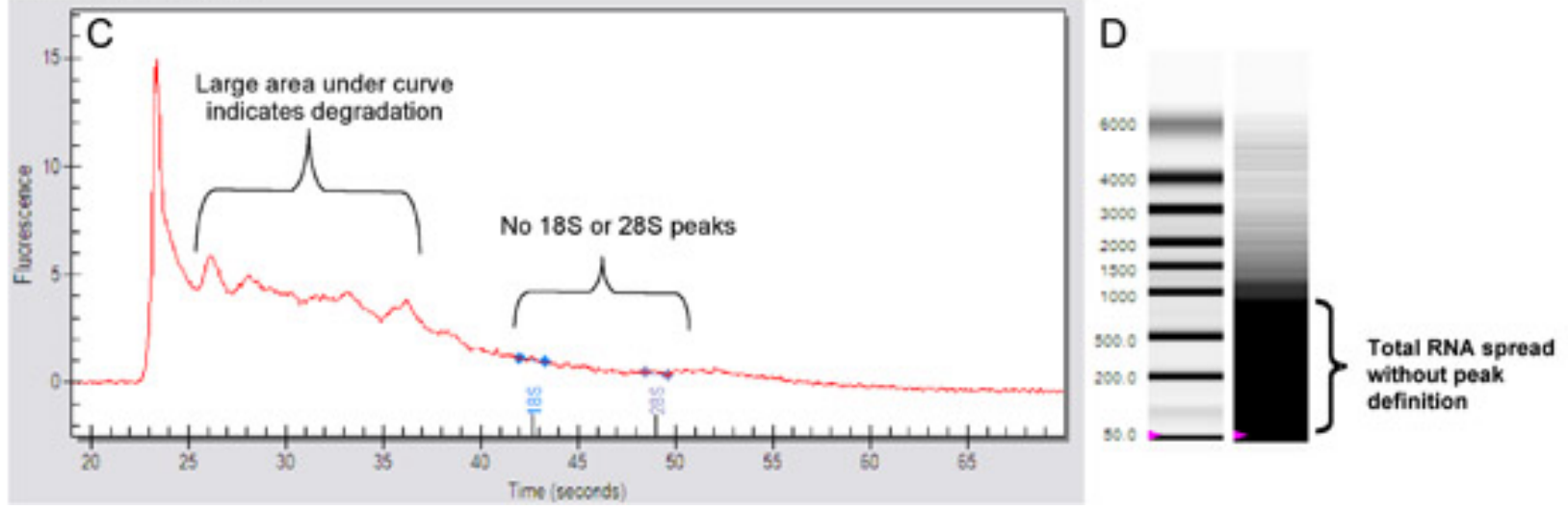

Figure 2: Total RNA quality control. $A+B$ ) Represents good quality total RNA after isolation, while $C+D$ ) illustrates what total RNA should not look like after isolation. A) An electropherogram with clear 18/28S peaks corresponding with the virtual gel in (B). C) An electropherogram showing a large area under the curve and no $18 \mathrm{~S} / 28 \mathrm{~S}$ peaks indicating RNA degradation. This is also shown in the virtual gel (D). 


\section{Figure 3: mRNA quality control}

\section{Good quality}
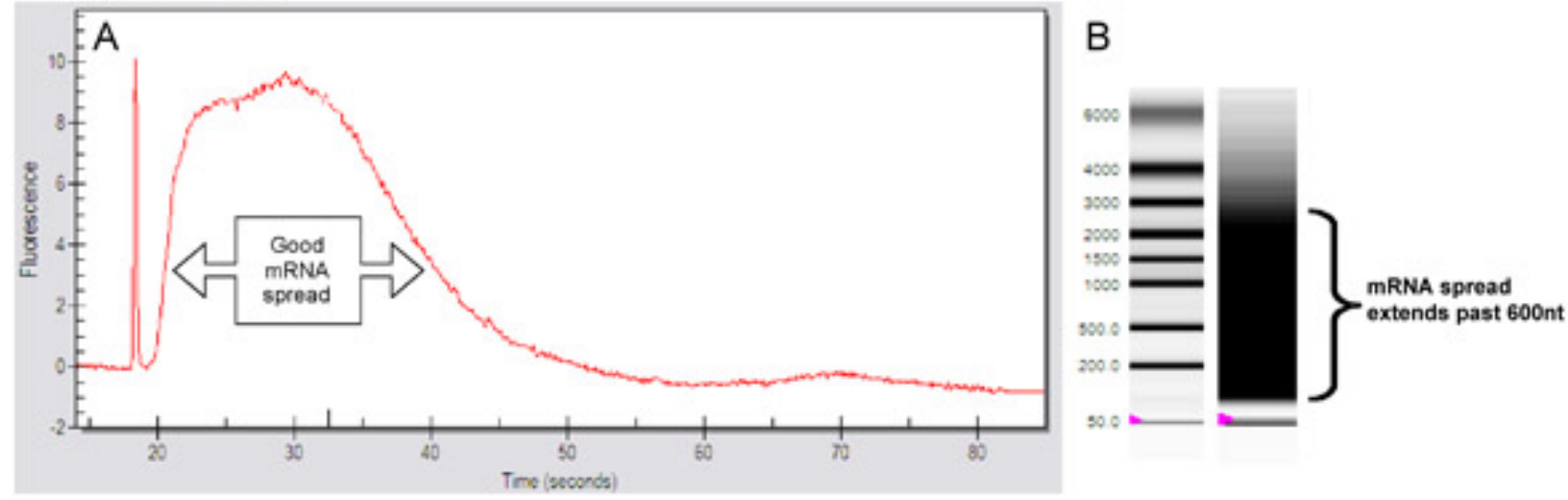

\section{Bad quality}
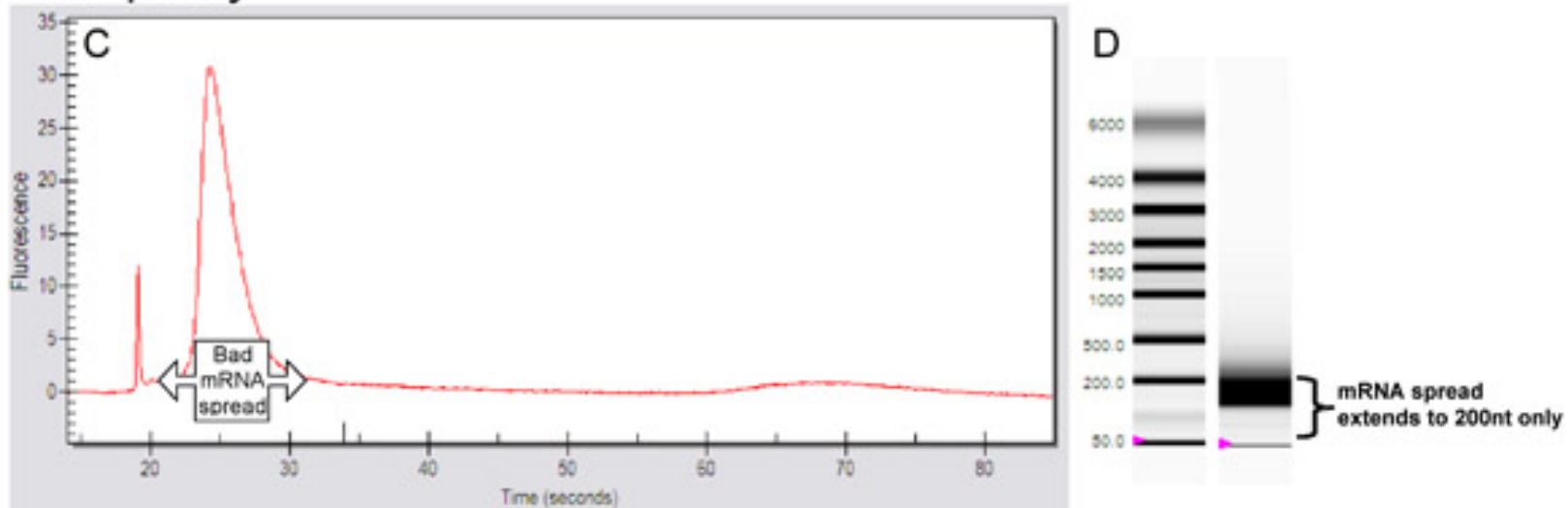

Figure 3: mRNA quality control. $A+B$ ) Electropherogram (A) and virtual gel (B) indicating the mRNA transcript length (spread) necessary for microarray studies, i.e extending past 600 nucleotides (nt). $C+D$ ) Electropherogram (C) and virtual gel (D) showing what an insufficient mRNA spread would look like, as the mRNA transcript length does not extend past 200nt.

\section{Discussion}

Here, we attempt to customize a protocol to extract homogeneous cell populations from heterogeneous postmortem brain tissue using the Arcturus XT system and related RNA extraction kits. As outlined above, we have made some modifications to the original protocols provided by the company, in order to maximize RNA integrity. The successful capturing of single cells depends solely on optimizing the steps prior to capture in order to achieve maximum cells with good RNA. These steps include the various parameters associated with tissue sectioning and staining. In short, with regards to tissue preparation these include: 1) decreasing the temperature gradient when sectioning, 2) only placing 2 sections per slide, 3) performing all dehydration steps on ice, 4) adding RNase Inhibitor to the stain, 5) increasing the dehydration duration in the final $100 \%$ ethanol to 3 minutes.

With regards to capturing, we feel that the combination of CapSure HS caps, with the software on Macro setting, should ensure optimal results. A humidity-controlled environment is critical to capturing single cells, as high humidity drastically reduces tissue "lift". High temperatures can also have a negative effect on RNA quality.

During the RNA isolation protocol with the PicoPure kit, there are two wash steps. It is important to check that all wash buffer is removed before transferring to another microcentrifuge tube for RNA elution, as any presence of the buffer in your final sample will result in less starting RNA for amplification. To ensure this, we centrifuge the final wash step for 2.5 minutes instead of 2 minutes as suggested in the protocol provided.

For the most part, the linear amplification protocol was according to the manufacturer's instructions. However, two crucial points should be taken into account when using this protocol: 1 ) try to limit loss of RNA via excess transferring of samples between tubes, by using only the $0.5 \mathrm{ml}$ tubes provided in conjunction with a $0.5 \mathrm{ml}$ block in the thermal cycler; and 2) when transferring the sample to the purification column for purification of cDNA, be sure to spin down the sample tubes after the first transfer. This will result in an additional 1-2ul of sample to add to the purification column and therefore can increase cDNA recovery. 
When making use of the TURBO end-labeling kit for biotin-labeling of your limited aRNA samples, we suggest adding an extra few $\mu$ l's of water before the centrifugation step, to increase the labeled RNA yield extracted from the column. An extra minute of centrifugation will also help in this regard, especially if you are working with degraded tissue, such as FFPE tissue.

We feel confident that this protocol will enable the user to isolate small homogeneous structures, such as single cell populations, within heterogenous postmortem brain tissue. This reduces dilution effects of surrounding structures and other cell-types located in the various layers within the brain, leading to results targeted towards the specific cell types under investigation. As the quality of the resulting RNA is good enough for microarray studies, we can begin to pinpoint specific molecular signatures of specific cell populations affected in disease states.

\section{Disclosures}

The production of this video-article was sponsored by Life Technologies.

\section{Acknowledgements}

We gratefully acknowledge Molecular Devices Analytical technologies/Arcturus for their generous donation of reagents used for the study. We also thank the Harvard Tissue Resource Center for providing postmortem human brain tissue. Funding: RO1MH76060 and P50MH080272.

\section{References}

1. Imbeaud, S., Graudens, E., Boulanger, V., Barlet, X., Zaborski, P., Eveno, E., Mueleer, O., Schroeder, A. \& Auffray, C. Towards standardization of RNA quality assessment using user-independent classifiers of microcapillary electrophoresis traces. Nucleic Acids Res. 33 , e56, (2005). 